\title{
Adaptive Update of Reference Capacitances in Conductive Fabric Based Robotic Skin
}

\author{
Takahiro Matsuno $^{1}$, Zhongkui Wang ${ }^{1}$, Kaspar Althoefer ${ }^{2}$ and Shinichi Hirai ${ }^{1}$
}

\begin{abstract}
This paper proposes a sensor using conductive fabric that can detect proximity and contact by measuring the capacitance between the sensor and the surrounding environment. Due to the flexibility of the sensor used, it can be easily integrated with industrial robot arms to monitor proximity and contact between the robot and the surrounding environment including humans for safety reasons. However, the surrounding environment is constantly changing and significantly affects the capacitance measurements. To apply such proximity sensors in this scenario, the environmental variations have to be considered and the influences on the capacitance measurements have to be eliminated to ensure stable and robust proximity measurements. Therefore, in this paper, we propose an approach to adaptively update the reference capacitance to eliminate the influence of the environment. To experimentally validate the proposed sensor and approach, we developed a twolink robot arm and embedded the proposed sensing technology with each link. Experimental results demonstrate that proximity and contact can be successfully detected by the proposed sensor, independently of whether the robot arm is at rest or moving in a potentially dynamic environment.
\end{abstract}

\section{INTRODUCTION}

Industrial robot arms have found many applications in many places especially in manufacturing. With the on-going trend of moving robots out of their cages and into the vicinity of humans, it becomes important to ensure the safety of humans working in those environments [1][2][3]. Previously, the safety of the human workers was ensured by physically separating the robot's working space from that of the humans [1][2][3]. Whilst in the past simple fences kept the robot apart from the human, today we see the introduction of a number of means to ensure that no harm comes to the human, including non-contact detection devices, such as a light curtain or a laser scanner [4], or contact-based detection devices, such as a pressure measurement mat or a bumper [5], avoiding any potentially harmful physical interaction between the machine and the workers. On the other hand, recent research works suggest that cooperative and interactive work between the robot and the worker has the potential to realize a more productive manufacturing process [6][7]. This, however, assumes that the operation areas of the robot and the human worker overlap, with potentially multiple collision points that could lead to injuries in the human worker. Since there is no physical boundary between the robot and the

\footnotetext{
${ }^{1}$ Takahiro Matusno, Zhongkui Wang and Shinichi Hirai are with the Department of Robotics, Ritsumeikan University, 1-1-1 Noji Higashi, Kusatsu, Shiga, Japan ma-tsunoefc/ wangzkefc/ hirai@se.ritsumei.ac.jp

${ }^{2}$ Kaspar Althoefer is with the Centre for Advanced Robotics @ Queen Mary, Faculty of Science \& Engineering, Queen Mary University of London, Mile End Rd, London E1 4NS, UK k. althoefer@qmul.ac.uk
}

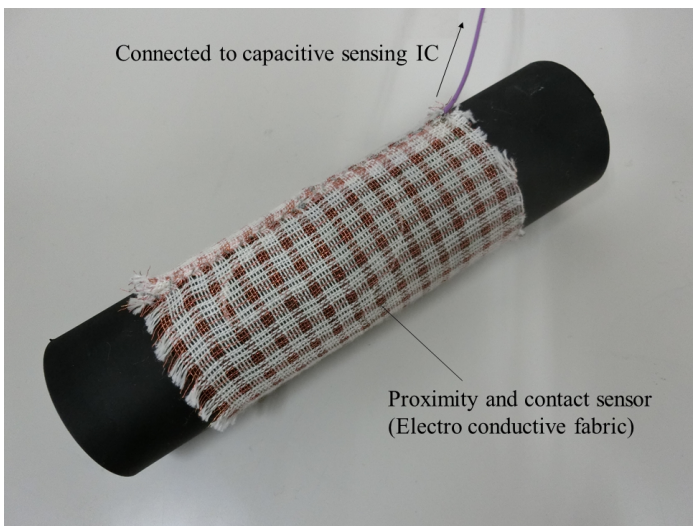

Fig. 1. Capacitance proximity and contact sensor using a conductive fabric.

worker in such a scenario, it is difficult to guarantee the safety of the worker employing the aforementioned methods.

In recent years, some research has aimed at developing robot arms capable of changing the stiffness of their links in an attempt to create articulated systems that can safely operate with a human in a human-robot interaction (HRI) scenario [8][9]. This new approach can ensure the worker's safety by automatically decreasing the stiffness, when the worker erroneously intrudes into the robot working area. In this context, a major challenge is to determine/detect the human intrusion or human proximity with respect to the articulated robot arm. In this paper, we propose the use of a sensorised robotic skin to measure the worker's vicinity with regards to the robot. Robotic skins operating as distributed sensors were studied in a number of research institutes; the principle of the sensorised skins is, in most cases, based on pressure sensors distributed across the outer skin of a robotic device [10][11], integrated force sensors [12], and electrical impedance tomography [13]. Although very suitable to measure contact, these methods are not capable of measuring proximity. Some recent research has put the focus on creating distributed proximity sensors that can be integrated with robot arms to obtain an indication on the distance between the worker and the robot, and, thus, improve the prediction of worker intrusion in the robot workspace [14][15][16]. With such a proximity sensing capability in place (measuring the relative distance between human and robot), it would be possible to adjust the stiffness of the robot links before contact with the human occurs, and render a potentially injury-inflicting collision into a 'soft interaction' considerably reducing the severity of the impact on the human. It is noted that most commercially available 
proximity sensors consist of rigid components and are, thus, difficult to be integrated with robot links whose stiffness can be controlled, without negatively impacting on the link stiffness by the sensor presence. Recent work has produced advancements in flexible proximity sensors with some research creating proximity sensors based on polydimethylsiloxane and copper meshes [17] or flexible circuit boards [18]. With our aim of not affecting the stiffness of the robot link through the introduction of our sensor, we propose here a distributed proximity sensor based on conductive fabric, which we have shown previously to successfully measure proximity, contact, and slippage [19][20]. Such proximity sensors [17][18][20] show excellent performances in static as well as dynamic scenarios. However, in a real working scenario, the surrounding environment, such as temperature, humidity, nearby objects, and robot dynamics, are constantly changing and these variations significantly affect the sensor signals, especially if the sensor measures proximity using the principles of capacitance measurements, as is the case for many proximity sensors. To apply such proximity sensors in a real working scenario, these environmental variations have to be considered and the influences on the capacitance measurements have to be eliminated to ensure stable and robust proximity measurements. Therefore, in this work, we propose an approach to adaptively update the reference capacitance eliminating the influences of environment variations. This is the main contribution of our paper.

The rest of the paper is organized as follows. Section II introduces the principle of proximity and contact sensor using conductive fabric, and the method of determining proximity and contact using the capacitance value. In section III, the update method of the reference capacitances for determining proximity and contact is presented. Experimental validation is given in Section IV. Section V concludes the paper.

\section{SENSOR PRINCIPLE}

\section{A. Concept}

The concept of the proximity and contact sensors is shown in Fig. 1. Our sensor skin is constructed using conductive fabric. Electrostatic capacity is generated between the electro-conductive fabric and any objects placed near the sensor. The value of electrostatic capacity can be measured by a capacitive sensing integrated circuit (IC). Due to the flexibility, the sensor with its shape-conforming characteristic can be easily integrated with a robot arm (Fig. 1), allowing the determination of proximity and contact.

Examples of measured electrostatic capacity are shown in Fig. 2 and 3. In this experiment, five different objects shown in Fig. 2 are approached to the proximity and contact sensor. In Fig. 2, each picture shows that the case of distance $50 \mathrm{~mm}$ between the object and the sensor. Measurement results of the capacitance against distance from the sensor are shown in Fig. 3. In Fig. 3, $x$ axis shows the distance between the sensor and object, and $y$ axis shows the capacitance of the sensor. According to the measurement results, the electrostatic capacity increases by the approaching object,

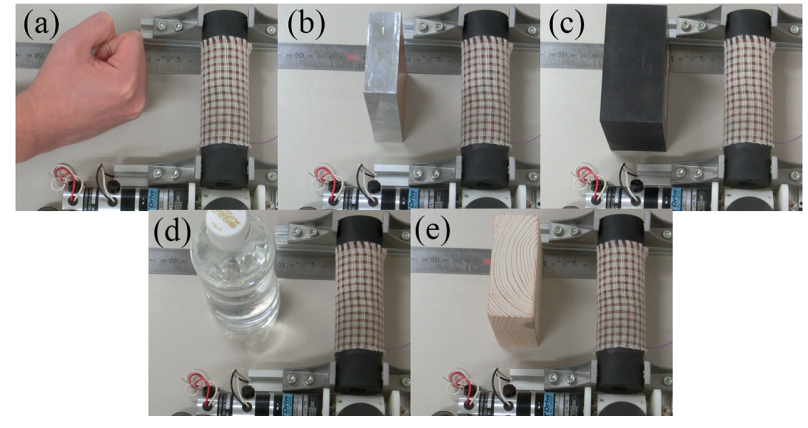

Fig. 2. Proximity experiment of (a)human hand, (b)aluminium object, (c)natural rubber, (d)water and (e) wood.

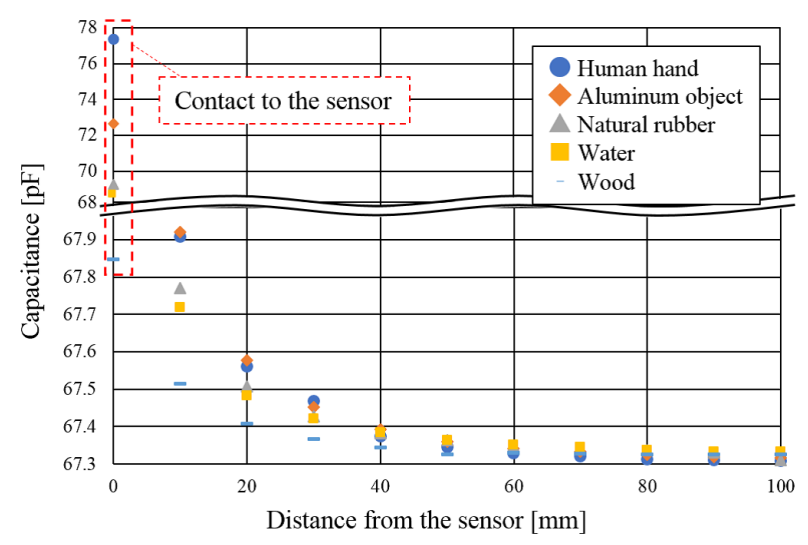

Fig. 3. Proximity distance and capacitance of the sensor.

and is again sharply altered when contact between the object and the sensor takes place.

\section{B. Evaluation method}

An example of measured electrostatic capacity is shown in Fig. 4. During the shown measurements, at first, between $0 \mathrm{~s}$ to $22.8 \mathrm{~s}$, no objects was placed close to the sensor; then, between 22.8 - $28.3 \mathrm{~s}$, a human hand approached the sensor. After $28.3 \mathrm{~s}$, the human hand has come in physical contact with the sensor.

Let $c_{k, i}$ be the most recent capacitance measurement of the $k$-th sensor, with variable $i$ representing the most recent measurement event. Each sensor measures the capacitance continuously, and $i$ is increasing along the measurement duration. In this study, when the environment around the $k$-th sensor is static, the sensor value is defined as reference capacitance $c_{k, i}^{\text {base }}$ for the $k$-th sensor. Using Fig. 4 as an example, we can see that the value in the range of $0 \mathrm{~s}$ to $22.8 \mathrm{~s}$ can be defined as reference capacitance. The following threshold values, $c_{k, i}^{\max }$ and $c_{k, i}^{\text {touch }}$ will be defined to judge if an object is approaching or getting into contact with the sensor. The proximity level is represented by a value between $0-1$ as shown in Fig. 5. The value $c_{k, i}^{\max }$ represents the electrostatic capacity of the $k$-th sensor, which is defined as the highest proximity level. The value $c_{k, i}^{\text {touch }}$ represents the electrostatic capacity of the $k$-th sensor, when an object got in contact with a sensor. Threshold values for the evaluation of proximity $c_{k, i}^{\max }$ and contact $c_{k, i}^{\text {touch }}$ of the $k$-th sensor are 


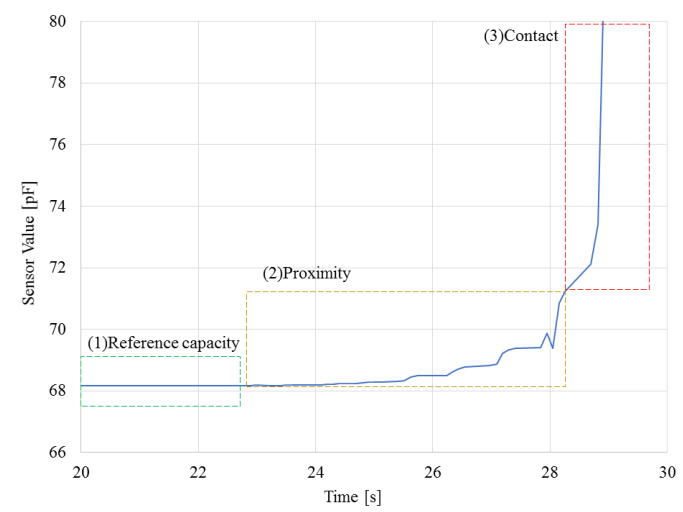

Fig. 4. Sensor value example of capacitance proximity and contact sensor.

derived from $c_{k, i}^{\text {base }}$. The threshold values are defined as

$$
\begin{gathered}
c_{k, i}^{\max }=g\left(c_{k, i}^{\text {base }}\right), \\
c_{k, i}^{\text {touch }}=h\left(c_{k, i}^{\text {base }}\right),
\end{gathered}
$$

where $g$ and $h$ are functions (determined empirically) for calculating the threshold values. First, contact to the $k$-th sensor is evaluated. Contact with the sensor occurs, if the latest measured value of electrostatic capacity satisfies

$$
c_{k, i}>c_{k, i}^{\text {touch }}
$$

If the measured value does not satisfy Eq. (3), our system assumes that no contact occurred and proximity is evaluated instead. The level of proximity is calculated as

$$
\text { Proximity }=\frac{c_{k, i}-c_{k, i}^{\text {base }}}{c_{k, i}^{\text {max }}-c_{k, i}^{\text {base }}} \text {. }
$$

By way of exception, if the value of Eq. (4) is over 1, the level of proximity is replaced with 1 as shown in Fig. 5. Proximity and contact to the $k$-th sensor are calculated by Eqs. (1) - (4). Then, measured data are logged as

$$
\mathbf{c}_{\boldsymbol{k}}=\left[c_{k, 1} c_{k, 2} \cdots c_{k, i}\right],
$$

where $c_{k, i}$ is the measured data of the $k$-th sensor at $i$-th time. Likewise, the joint angles of the robot arm are measured and

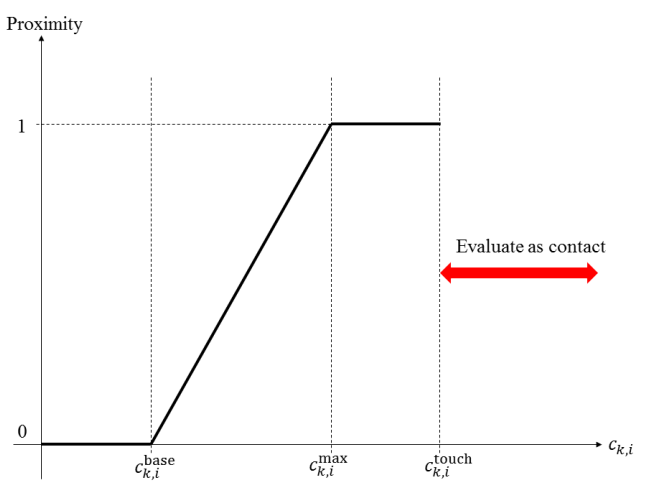

Fig. 5. Definition of proximity level. logged. For a robot with $L$ joints, angle of the $l$-th joint at $i$-th time is represented by $\theta_{l, i}$, and the data are logged as

$$
\boldsymbol{\Theta}_{\boldsymbol{i}}=\left[\theta_{1, i} \theta_{2, i} \ldots \theta_{l, i} \ldots \theta_{L, i}\right]^{T},
$$

where $\theta_{l, i}$ is the angle of the $l$-th joint measured at $i$-th time. If the reference capacitance $c_{k, i}^{\text {base }}$ can be determined as one value, the levels of proximity and contact can be determined with these proposed equations. However, the reference capacitance $c_{k, i}^{\text {base }}$ can easily change due to changes in the environment or the robot arm's pose. Therefore, the reference capacitance of each sensor must be updated when the environment or arm pose are changing.

\section{Method to UPDATE THE REFERENCE CAPACITANCE}

We consider two fundamental scenarios in this research: (1) the robot arm is at a standstill, and (2) the robot arm is moving. The methods of updating the reference capacitance are presented in detail for each scenario.

\section{A. Standstill case}

In this case, the measured capacitance data between the $(i-n)$-th and $i$-th were selected as shown in Fig. 6. The average value of the selected data was calculated by Eq. (7) and is represented as $c_{k, i}^{\text {avg }}$

$$
c_{k}^{\mathrm{avg}}=\frac{1}{n} \sum_{m=i-n}^{i} c_{k, m} .
$$

Then, the standard deviation of the selected data is calculated as

$$
s=\sqrt{\frac{1}{n-1} \sum_{m=i-n}^{i}\left(c_{k, m}-c_{k}^{\mathrm{avg}}\right)^{2}} .
$$

Finally, the standard deviation $s$ is evaluated by

$$
s<\alpha,
$$

where $\alpha$ is the threshold value to trigger a reference capacitance update, defined empirically. If the standard deviation of the selected data satisfies Eq. (9), the average value of the selected data as shown in Eq. (7) is used as a new reference capacitance $c_{k, i}^{\text {base }}$. When the environment changes gradually,

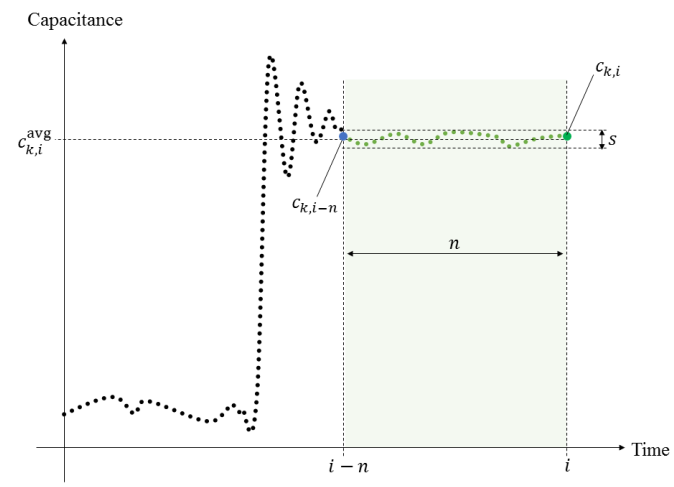

Fig. 6. Method to update the reference capacitance in standstill scenarios 


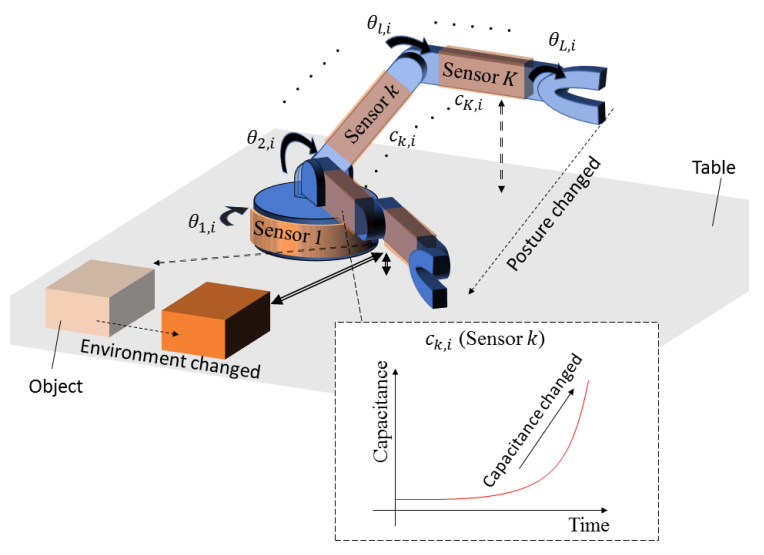

Fig. 7. Reference capacitance changing in working scenario.

or it becomes stable after a change, the standard deviation $s$ will decrease. On the other hand, when a human hand or body approaches, the sensor value will be not stable and the standard deviation of the selected data will increase. Therefore, if the threshold value $\alpha$ is defined adequately, this method is able to trigger the update of the reference capacitance.

\section{B. Dynamic case}

In this case, the environment is defined as containing a number of objects like a wall, table or frame in the vicinity of the robot arm. When the robot arm is moving, the distances between the sensors and the objects placed in environment are constantly changing, therefore the electrostatic capacitances of the sensors are not static during these robot arm movements as shown in Fig. 7. In this case, the reference capacitance of each sensor needs to be adjusted depending on the robot arm pose. In this method, we assume that the initial state of environment is fixed and the environment will be kept in its initial state for a few seconds after the robot arm has started moving. Each sensor value and each joint angle of the robot links are measured repeatedly and the obtained sensor signals are evaluated, i.e., the approximated function of relationship between the capacitance of $k$-th sensor and the robot arm pose is computed. For computing, first, the logged data between $i_{s}$ to $i$ are selected. Variable $i_{s}$ represents the starting time of the data for fitting. In the case of first time fitting of the approximated function, $i_{s}$ is set to 0 . Then, $c_{k, i_{s}}$ to $c_{k, i}$ and $\boldsymbol{\Theta}_{\boldsymbol{i}_{\boldsymbol{s}}}$ to $\boldsymbol{\Theta}_{\boldsymbol{i}}$ are fitted by a function, which function was determined empirically. The fitted function is represented by $f_{k ; i_{s}, i}$, and this function shows relationship between the capacitance of $k$-th sensor and the robot arm pose. The reference capacitance of the $k$-th sensor is updated by

$$
c_{k, i}^{\mathrm{base}}=f_{k ; i_{s}, i}\left(\boldsymbol{\Theta}_{\boldsymbol{i}}\right) .
$$

Then, proximity to and contact with the $k$-th sensor are calculated by Eqs. (1) - (4) using the reference capacitance.

If the environment condition remains unchanged, the function $f_{k ; i_{s}, i}$ will not have to be updated. However, when the environment condition experiences a change, the function $f_{k ; i_{s}, i}$ will need to be updated. To determine the timing of the update, the latest measured data $c_{k, i}$ is evaluated with fitted function $f_{k ; i_{s}, i}$ as

$$
\left|c_{k, i}-f_{k ; i_{s}, i}\left(\boldsymbol{\Theta}_{\boldsymbol{i}}\right)\right|>\beta,
$$

where $\beta$ is the threshold value to trigger the update; the threshold value has been defined empirically. If the latest measured data satisfy Eq. (11) at $i$-th time, the number of $i$ is saved as

$$
\begin{aligned}
& i_{e}^{-}=i-\epsilon, \\
& i_{e}^{+}=i+\epsilon,
\end{aligned}
$$

where $\epsilon$ represents the offset value avoiding instability in values. Then, the measured data is divided into two data sets; previous data and new data. Previous data are measured from $i_{s}$ to $i_{e}^{-}$as shown in Fig. 8. New data are measured from $i_{e}^{+}$ to $i$. By using these two data sets, the approximated functions of previous and new data are refitted as shown in Fig. 9. The fitted function of the relationship between the capacitance and the robot arm pose from $i_{s}$ to $i_{e}^{-}$is represented by $f_{k ; i_{s}, i_{e}^{-}}$, and after $i_{e}^{+}$to $i$ is represented by $f_{k ; i_{e}^{+}, i}$. Then, each function is evaluated. First, the error of latest measured capacitance $c_{k, i}$ and the fitted function are calculated. Errors of functions $f_{k ; i_{s}, i_{e}}$ and $f_{k ; i_{e}^{+}, i}$ are calculated as

$$
\begin{gathered}
\delta_{k ; i_{s}, i_{e}^{-}}=f_{k ; i_{s}, i_{e}^{-}}\left(\boldsymbol{\Theta}_{\boldsymbol{i}}\right)-c_{k, i}, \\
\delta_{k ; i_{e}^{+}, i}=f_{k ; i_{e}^{+}, i}\left(\boldsymbol{\Theta}_{\boldsymbol{i}}\right)-c_{k, i},
\end{gathered}
$$

where $\delta_{k ; i_{s}, i_{e}^{-}}$and $\delta_{k ; i_{e}^{+}, i}$ represent the error of fitted function $f_{k ; i_{s}, i_{e}^{-}}$and $f_{k ; i_{e}^{+}, i}$, respectively. Let us compare each function $f_{k ; i_{s}, i_{e}^{-}}$and $f_{k ; i_{e}^{+}, i}$ by using evaluation points $p_{k ; i_{s}, i_{e}^{-}}$, $p_{k ; i_{e}^{+}, i}$ and $p_{k_{\text {draw }}}$ which represent the score of $f_{k ; i_{s}, i_{e}^{-}}$, score of $f_{k ; i_{e}, i}$ and the score for when neither function satisfies the evaluation conditions. First, the fitted function $f_{k ; i_{s}, i_{e}^{-}}$is evaluated by

$$
\begin{gathered}
\left|\delta_{k ; i_{e}^{+}, i}\right|>\left|\delta_{k ; i_{s}, i_{e}^{-}}\right|, \\
\beta>\left|\delta_{k ; i_{s}, i_{e}^{-}}\right| .
\end{gathered}
$$

If $\delta_{k ; i_{s}, i_{e}^{-}}$satisfies both Eq. (16) and (17), score of $f_{k ; i_{s}, i_{e}^{-}}$ is obtained as

$$
p_{k ; i_{s}, i_{e}^{-}}=p_{k ; i_{s}, i_{e}^{-}}^{\prime}+1,
$$

where $p_{k ; i_{s}, i_{e}^{-}}^{\prime}$ represents the previous score of $f_{k ; i_{s}, i_{e}^{-}}$. Then, the fitted function $f_{k ; i_{e}^{+}, i}$ is evaluated as

$$
\begin{gathered}
\left|\delta_{k ; i_{s}, i_{e}^{-}}\right|>\left|\delta_{k ; i_{e}^{+}, i}\right|, \\
\beta>\left|\delta_{k ; i_{e}^{+}, i}\right| .
\end{gathered}
$$

Likewise, if $\delta_{k ; i_{e}^{+}, i}$ satisfies both Eq. (19) and (20), the score of $f_{k ; i_{e}^{+}, i}$ is obtained as

$$
p_{k ; i_{e}^{+}, i}=p_{k ; i_{e}^{+}, i}^{\prime}+1
$$

where $p_{k ; i_{e}^{+}, i}^{\prime}$ represents the previous score of $f_{k ; i_{e}^{+}, i}$. If both functions $f_{k ; i_{s}, i_{e}^{-}}$and $f_{k ; i_{e}^{+}, i}$ do not satisfy these conditions, $p_{k_{\text {draw }}}$ will get a score as follows

$$
p_{k_{\mathrm{draw}}}=p_{k_{\mathrm{draw}}}^{\prime}+1
$$


TABLE I

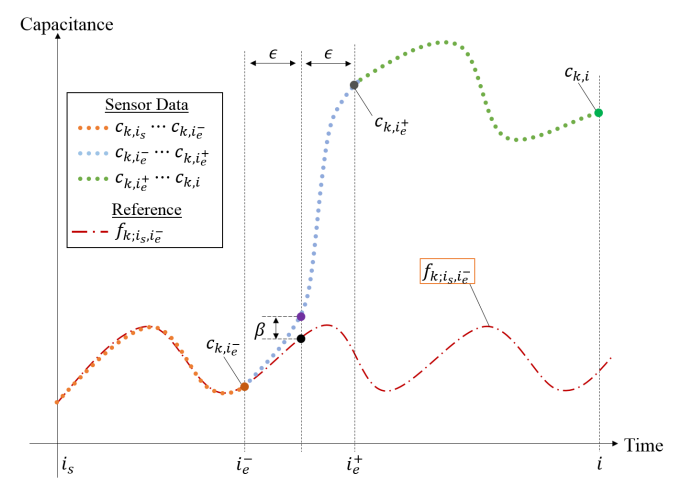

Fig. 8. Update method of the reference capacitance in the dynamic scenario.

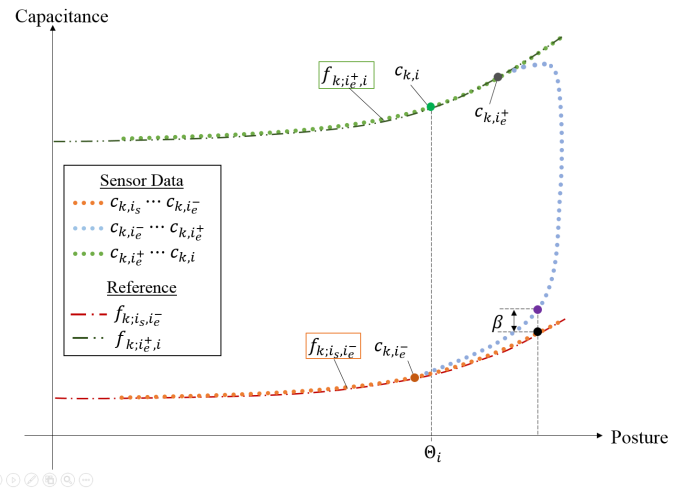

Fig. 9. Relationship between robot arm posture and capacitance in working scenario.

where $p_{k_{\text {draw }}}^{\prime}$ represents the previous value of $p_{k_{\text {draw }}}$. Every time the $k$-th sensor measures new data of $c_{k, i}$. These evaluations are repeated and each score $p_{k ; i_{s}, i_{e}^{-}}, p_{k ; i_{e}^{+}, i}$ and $p_{k_{\mathrm{draw}}}$ will be increased. Then, each score values are compared to determine which value exceeds the threshold value $\gamma$ first. If the score $p_{k ; i_{s}, i_{e}^{-}}$exceeds the threshold value $\gamma$ first, the fitted function $f_{k ; i_{s}, i_{e}^{-}}$is used to describe the relationship between capacitance and robot arm pose. On the other hand, if the score $p_{k ; i_{e}^{+}, i}$ exceeds the threshold value $\gamma$ first, the fitted function $f_{k ; i_{e}, i}$ is used for the function relationship between capacitance and robot arm pose. In this case, the value $i_{s}$ is set as $i_{e}^{\prime}$. Finally, if neither function satisfies the evaluation conditions and the score $p_{k_{\text {draw }}}$ exceeds the threshold value $\gamma$, the data measured after $i_{e}^{-}$-th time is ignored. The time is saved as $i_{e}^{++}=i$, and a new fitted function $f_{k ; i_{e}^{++}, i}$ will be evaluated with $f_{k ; i_{s}, i_{e}^{-}}$using the presented method. When the environment is stable again after a change had occurred, the error of latest measured capacitance $c_{k, i}$ and the fitted function of $f_{k ; i_{e}^{+}, i}$ will be smaller. In this case, the score $p_{k ; i_{e}^{+}, i}$ would exceed the threshold value first, and the fitted function $f_{k ; i_{e}^{+}, i}$ is used to calculate the reference capacitance. On the other hand, when any object approaches the sensor temporarily and then the environment returns to the initial state, the error of latest measured capacitance $c_{k, i}$ and the fitted function of $p_{k ; i_{s}, i_{e}}$ will be smaller. In this case, the
THRESHOLD VALUES FOR EVALUATION OF PROXIMITY AND CONTACT. THE UNIT OF $n, \gamma$ AND $\epsilon$ ARE MEASUREMENT CYCLES.

\begin{tabular}{cccccc}
\hline Threshold & $n$ & $\alpha[\mathrm{pF}]$ & $\beta[\mathrm{pF}]$ & $\gamma$ & $\epsilon$ \\
\hline Values & 100 & 0.5 & 0.5 & 50 & 10 \\
\hline
\end{tabular}

score $p_{k ; i_{s}, i_{e}^{-}}$would exceed the threshold value first, and the fitted function $f_{k ; i_{s}, i_{e}^{-}}$is used to calculate the reference capacitance. With these methods, the reference capacitance and the function for calculating the reference capacitance are updated automatically.

\section{EXPERIMENTAL VALIDATION}

\section{A. Experimental setup}

In this section, the proposed capacitance-based proximity and contact sensor is experimentally validated. The robot arm for the experiment is shown in Fig. 10. The robot has two links and one degree of freedom. In our study the links are made from rubber and have a length of $150 \mathrm{~mm}$. As shown in Fig. 10, the two links are referred to as Link1 and Link2. Each link is equipped with our proposed sensor as shown in Fig. 10. Sensor number is 2, and joint number $L$ is 1 . The sensors are fabricated by electro conductive fabric (DC6006, Shibata Technotex Co. LTD., Japan), and electrostatic capacitance is measured using an integrated circuit (FDC2241, Texas Instruments, U.S.A). The measured values are sent to the PC, and the values are used for evaluation of proximity and contact. The evaluation method was programmed in MATLAB, and Eqs. (1)-(2) are defined as

$$
\begin{gathered}
c_{k, i}^{\max }=c_{k, i}^{\text {base }}+0.5 \mathrm{pF}, \\
c_{k, i}^{\text {touch }}=c_{k, i}^{\text {base }}+1.0 \mathrm{pF} .
\end{gathered}
$$

Threshold values for evaluation of proximate and contact are given in Table I. The sampling time of measurement is about $0.15 \mathrm{~s}$. The relationship between capacitance and robot arm pose is defined by a 4th-order polynomial function. The coefficients of the fitted polynomial function are calculated by curve fitting using MATLAB.

The first experiment was performed to validate the proximity and contact sensing capabilities of our sensor. The validation scenario used for proximity and contact sensing is shown in Fig. 11 (a). A human hand approaches the sensor with a low speed, and makes contact at last. The sensing states of the proximity or contact are compared with actual states, then the sensing ability is validated. This validation is done for each link. Experiments are performed to validate if the proposed method is able to trigger the update of the reference capacitance. The experiment setup is shown in Fig. 11 (b). An aluminium block is placed near the robot arm. In this situation, the environment was changed; however, the change was only temporarily since the block remains statically in its position. This validation is performed for each link in the same way. These validations are carried out for 
each case of the standstill scenario and the dynamic scenario of the robot arm.

\section{B. Experimental results}

1) Evaluation of proximity and contact determination in the standstill case: Experimental results of proximity and contact of each sensors are represented. The capacitance measured by the sensor of Link1 is shown in the Fig. 12 (a), and the result measured by the sensor of Link 2 is shown in the Fig. 12 (c). In Fig. 12 (a), the reference capacitance was $68.21 \mathrm{pF}$ and it was based on the measurement result before $46.5 \mathrm{~s}$. After that, the human hand approached the Link1 from $46.5 \mathrm{~s}$ to $55.6 \mathrm{~s}$. At this time, the system estimated that there is a proximity, and the extent of its proximity was indicated by using the thresholds shown in Table I. At a proximity of 0 , the human hand is about $5 \mathrm{~cm}$ away from Link1, and at a proximity of 1 , the human hand is almost touching the sensor. After $55.6 \mathrm{~s}$, the human hand was contacting the sensor of Link1 directly. The system estimated contact with the sensor using the threshold shown in Table I. In this experiment, it was confirmed that the system worked as anticipated without erroneously updating the reference capacitance.

Likewise, the system succeeded to estimate the proximity and occurrence of contact of the sensor of Link 2 as shown in Fig. 12 of (c). The reference capacitance was $68.22 \mathrm{pF}$ and it was based on the measurement result before $23.8 \mathrm{~s}$. The human hand approached Link 2 from $23.8 \mathrm{~s}$ to $32.1 \mathrm{~s}$, and after $32.1 \mathrm{~s}$, the hand got into contact with the sensor. At the same time, the system determined correctly that an object had been approaching the sensor by flagging first proximity and subsequently contact; the system correctly kept the reference capacitance unchanged.

2) Update of the reference capacitance in the standstill case: This experimental study aims to validate whether our method can trigger the update for the reference capacitance correctly. In this experiment, an aluminium object was placed near the robot arm which was at a standstill. The experimental results of the sensor integrated with Link1 are shown in Fig. 12 (b). In this experiment, the environment was first changed and then fixed at $98.2 \mathrm{~s}$. At $112.5 \mathrm{~s}$, the measured electrostatic capacitance value satisfied Eq. (9), and the system updated the electrostatic capacitance measured after

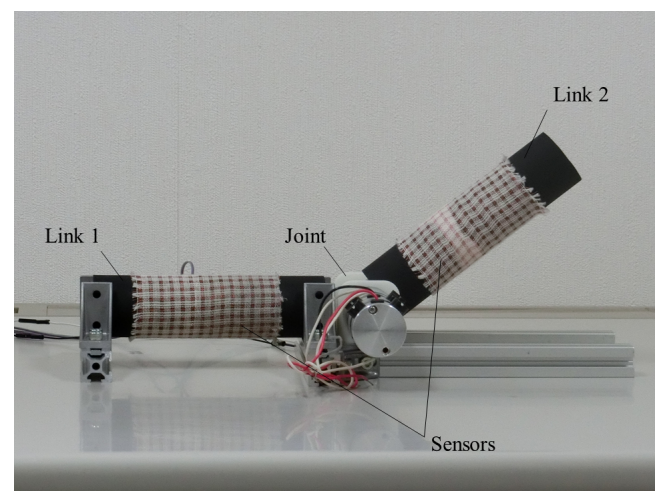

Fig. 10. Experimental setup for sensor validation.
98.2 $\mathrm{s}$ as a new reference value. The results of the sensor on Link2 are shown in Fig. 12 (d). The measured electrostatic capacitance value satisfied Eq. (9) at $83.3 \mathrm{~s}$, and the system updated the electrostatic capacitance measured after $68.9 \mathrm{~s}$ as a new reference value. From these experiments, it was confirmed that the proposed method can update the reference capacitance when there are environmental changes in the vicinity of the robot arm.

3) Evaluation of proximity and contact determination in the dynamic case: Experimental results of proximity and contact sensing in a dynamic scenario of the robot arm are represented. The capacitance measured by the sensor of Link1 is shown in Fig. 13 (a), and the results measured by the sensor of Link2 are shown in Fig. 13 (d). From the results, it can be confirmed that the capacitance values were fluctuating (unstable) in the case of a dynamic scenario. The measured results of relationship between capacitance and robot arm pose are shown in Fig. 13 (c) and (f). 4th-order polynomial functions were fitted to the measured results of Fig. 13 (c) and (f), and fitted functions were used to calculate the reference capacitance. In these experiments, the initial state of the environment is fixed and the environment is kept a few seconds after the robot arm starts moving.

In Fig. 13 (a), the reference capacitance, shown as a dashed orange line, is calculated from the fitted function of Fig. 13 (c). The human hand approached and got into contact with Link1 several times. In every case, the system correctly predicted that there were occurrences of proximity and contact. Also, there were no false alarms when there was nothing approaching or contacting the sensor of Link1.

In the case of Link2 as shown in Fig. 13 (d), the measured capacitance changed more than was the case for Link1 because of the movement of the robot arm. However, the system estimated occurrences of proximity and contact correctly using the reference capacitance which were calculated from fitted functions, as shown in Fig. 13 (f). Likewise, the system did not erroneously estimate occurrences of proximity and contact when the hand was not approaching Link2.

These experiments confirm that the proposed methods can estimate the occurrences of proximity and contact even if the robot arm is moving.

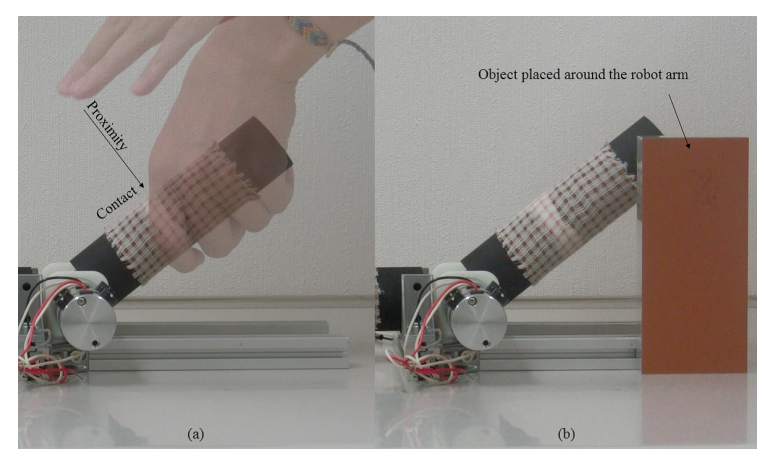

Fig. 11. Experimental setup for validation of (a) proximity and contact sensing and (b) reference value updating. 

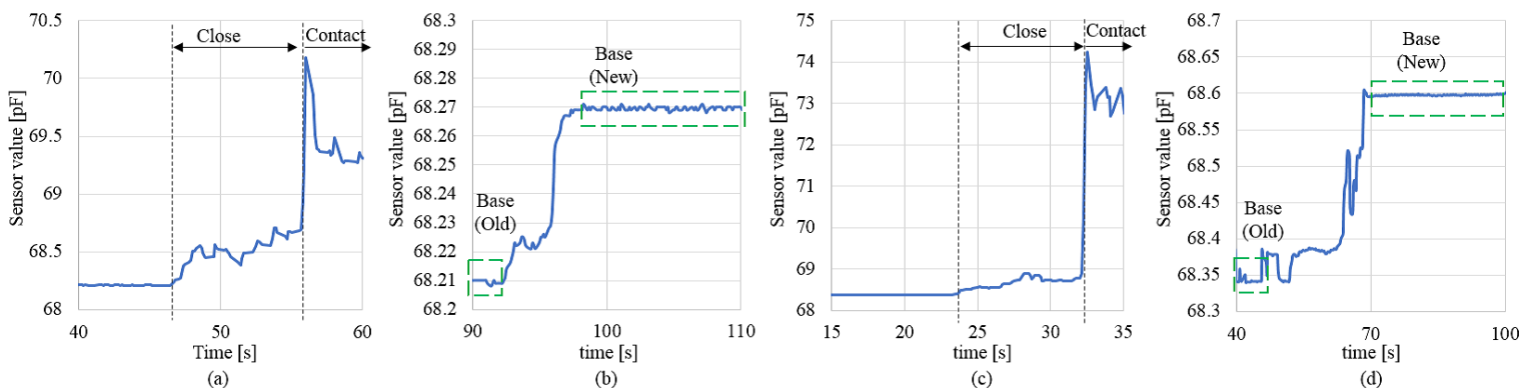

Fig. 12. Experimental results when the robot arm is standstill. (a)Proximity and contact evaluation of Link1, (b)The reference capacitance update of Link1, (c)Proximity and contact evaluation of Link2 and (d)The reference capacitance update of Link2.
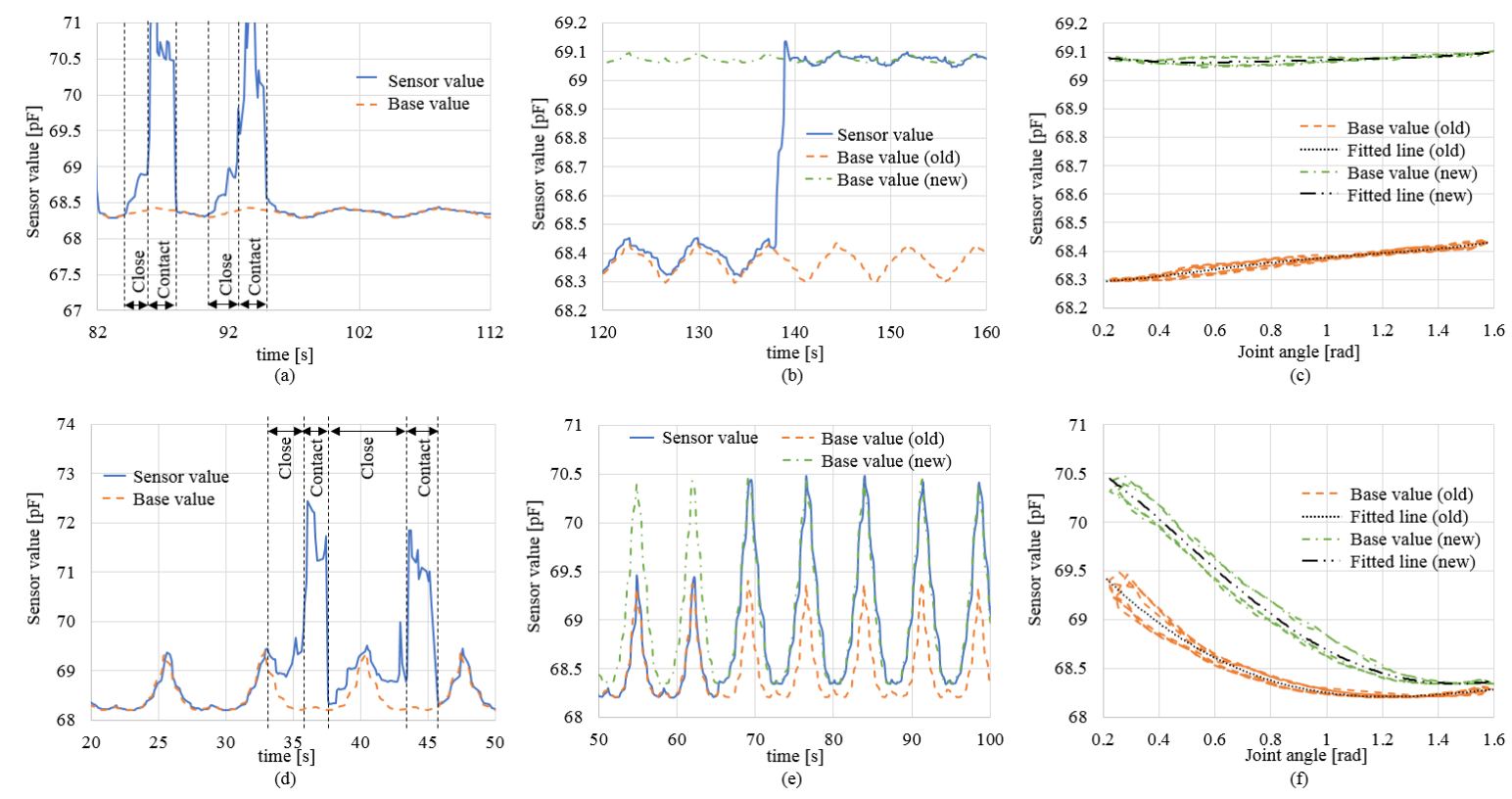

Fig. 13. Experimental results when the robot arm is working. (a)Proximity and contact evaluation of Link1, (b)The reference capacitance update of Link1, (c)Relationship between capacitance and robot arm posture of Link1, (d)Proximity and contact evaluation of Link2, (e)The reference capacitance update of Link2 and (f)Relationship between capacitance and robot arm posture of Link2.

4) Update of the reference capacitance in the dynamic case: These final experiments validate that the method can trigger the update of the reference capacitance, as required. Here, the aluminium blocks are placed outside the motion range of the robot arm to change the environment around the robot arm. The experimental results for the sensors on Link1 and Link2 are shown in Fig. 13 (b) and (e). The measured results of relationship between capacitance and robot arm pose are shown in Fig. 13 (c) and (f) with dot-dashed green lines. Again, 4th-order polynomial functions were fitted to the measured results as shown with black lines in Fig. 13 (c) and (f).

When the environment changed, the latest measured capacitance satisfies Eq. (11), and the system started to divide measurement data into previous data set and new data set. Function $f_{k ; i_{s}, i_{e}^{-}}$was fitted to the set of previously measured data, and function $f_{k ; i_{e}^{+}, i}$ was fitted to the set of newly measured data. The error of both fitted function is evaluated. The score of the function $f_{k ; i_{e}^{+}, i}$ is larger than $\gamma$ first, and the relationship between capacitance and robot arm pose is updated. These are confirmed in both sensors on Link1 and Link2. The reference capacitance of each link calculated by the updated function is shown as green lines in Fig. 13 (b) and (e). From these experiments, it was confirmed that the proposed method can update the function of relationship between capacitance and robot arm pose when the environment around the robot arm changes.

\section{CONCLUSIONS}

In this study, we proposed a proximity and contact sensor using conductive fabric. Since the proposed sensor consists of a flexible conductive fabric, the sensor has flexibility and it can be applied to soft and stiffness-controllable robot links greatly improving the safety of humans.

In the proposed sensor, the capacitance generated between the conductive fabric and the environment is measured. The proximity between the user and the sensor as well as the occurrence of contact are estimated using the measured capac- 
itance. The sensor's capacitance value when confronted with an obstacle-free environment (i.e., no object is approaching the sensor) is defined as the reference capacitance. The measured capacitance is then compared with the reference capacitance to estimate proximity between the sensor and the user as well as occurrences of contact. Since the reference capacitance changes as the environment around the robot arm changes, it is necessary to continuously update the reference capacitance while using this sensor. We distinguish between two main cases: (1) the robot arm is at standstill and (2) the robot arm is moving (dynamic scenario); two methods of updating the reference capacitance are proposed.

When the robot arm is at a standstill, the measured capacitance data between $(i-n)$-th to $i$-th sampling time are selected. If the standard deviation of the selected data falls under the threshold value, the average value of selected data is taken as a new reference capacitance.

When the robot arm is moving, the reference capacitance differs for each pose of the robot arm. For this reason, a relationship between capacitance and robot arm pose is obtained from the measured capacitance and the joint angles of the robot arm. The reference capacity is calculated from a function fitting the relationship between capacitance and robot arm pose. When the measured capacity largely deviates from the fitted function, the measurement data is split into previous data and new data. Then, two functions were fitted to each data set. For evaluation, the error of latest measured capacitance and the fitted function are calculated. The error of both fitted function is evaluated, and the better one is used as the function for further processing. The reference capacitance is then recalculated by the selected function.

To validate the proposed sensor and its prediction capabilities, a 2-link robotic arm equipped with the proposed sensor was created. The capacitance of the conductive fabric was measured, and the proximity and contact were estimated by the proposed method. It was confirmed that proximity and contact can be identified when the robot arm is at a standstill. The reference capacitance was updated by our algorithm when the environment was changed near the robot arm. In the same way, a second experiment was conducted in a dynamic scenario, and it was confirmed that proximity and contact prediction was also possible. When the robot arm was moving, the distances between the sensors and the objects placed in environment are constantly changing, therefore the electrostatic capacitances of the sensors are constantly changing, too. By updating the reference capacitance for each pose of the robot arm, out algorithm succeeded to detect proximity and contact irrespectively of the pose of the arm.

The final goal of this research is to ensure the safety of humans working with this sensor. To realize that, the control method of robot arm to avoid contacting with human is necessary. Therefore, we will study the robot control method for human safety using this sensor in the future.

\section{ACKNOWLEDGMENT}

This work was supported by JSPS KAKENHI Grant Number JP18K13734.

\section{REFERENCES}

[1] ISO 12100-2:2003, Safety of machinery - Basic concepts, general principles for design - Part 2: Technical principles.

[2] H. William, "Industrial safety handbook," London : McGraw, 1977.

[3] W. B. Creighton and N. Gunningham, "The Industrial relations of occupational health \& safety," Sydney, NSW : Croom Helm Australia, 1985.

[4] G. Pope, L. M. Lerjen, S. Mullener, S. Schlapfer, T. Walti, J. Widmer and C. Studer, "Light curtain localization via compressive sensing," in Proceeding IEEE International Conference on Acoustics, Speech and Signal Processing, pp 2558-2562, Canada, May 26-31, 2013.

[5] S. Dabrowski and Z. Branski, "Pressure Sensitive Mats as Safety Devices in Danger Zones," International Journal of Occupational Safety and Ergonomics, vol. 4, No. 4, pp. 499-519, 1998.

[6] O. Khatib, K. Yokoi, O. Brock, K. Chang and A. Casal, "Robots in Human Environments: Basic Autonomous Capabilities," The International Journal of Robotics Research, vol. 18, Issue 7, pp. 684-696, 1999.

[7] S. Haddadin, A. Albu-Schaffer and G. Hirzinger, "Requirements for Safe Robots: Measurements, Analysis and New Insights," The International Journal of Robotics Research, vol. 28, Issue 11-12, pp. $1507-1527,2009$.

[8] A. Stilli, L. Grattarola, H. Feldmann, H. A. Wurdemann and K. Althoefer, "Variable Stiffness Link (VSL): Toward inherently safe robotic manipulators," in Proceeding IEEE International Conference on Robotics and Automation, pp 4971-4976, Singapore, May 29 June 2, 2017.

[9] A. Stilli, H. A. Wurdemann and K. Althoefer, "A Novel Concept for Safe, Stiffness-Controllable Robot Links," Soft Robotics, vol. 4, No. 1, pp 16-22, 2017.

[10] P. Maiolino, M. Maggiali, G. Cannata, G. Metta and L. Natale, "A Flexible and Robust Large Scale Capacitive Tactile System for Robots," IEEE Sensors Journal, vol. 13, Issue 10, pp 3910-3917, 2013.

[11] M. Hoffmann, Z. Straka, I. Farkas, M. Vavrecka, and G. Mettae, "Robotic Homunculus: Learning of Artificial Skin Representation in a Humanoid Robot Motivated by Primary Somatosensory Cortex," IEEE Transactions on Cognitive and Developmental Systems, vol. 10, Issue 2, pp 163-176, 2017.

[12] M. Kaboli, D. Feng, K. Yao, P. Lanillos and G. Cheng, "A TactileBased Framework for Active Object Learning and Discrimination using Multimodal Robotic Skin," IEEE Robotics and Automation Letters, vol. 2, Issue 4, pp 2143-2150, 2017.

[13] D. Silvera-Tawil, D. Rye, M. Soleimani and M. Velonaki, "Electrical Impedance Tomography for Artificial Sensitive Robotic Skin: A Review," IEEE Sensors Journal, vol. 15, Issue 4, pp 2001-2016, 2015.

[14] Y. Ye, C. He, B. Liao and G. Qian, "Capacitive Proximity Sensor Array With a Simple High Sensitivity Capacitance Measuring Circuit for Human-Computer Interaction,” IEEE Sensors Journal, vol. 18, Issue 14, pp 5906-5914, 2018.

[15] Y. S. Kim, S. I. Cho, D. H. Shin, J. Lee and K. H. Baek, "Single Chip Dual Plate Capacitive Proximity Sensor With High Noise Immunity," IEEE Sensors Journal, vol. 14, Issue 2, pp 309-310, 2014.

[16] D. Goeger, M. Blankerts and H. Woern, "A tactile proximity sensor," SENSORS, pp 589-594, USA, Nov. 1-4, 2010.

[17] H. K. Lee, S. I. Chang and E. Yoon, "Dual-Mode Capacitive Proximity Sensor for Robot Application: Implementation of Tactile and Proximity Sensing Capability on a Single Polymer Platform Using Shared Electrodes," IEEE Sensors Journal, vol. 9, Issue 12, pp 1748-1755, 2009.

[18] S. Tsuji and T. Kohama, "Tactile and proximity sensor using selfcapacitance measurement on curved surface," in Proceeding IEEE International Conference on Industrial Technology, pp 934-937, Canada, March 22-25, 2017.

[19] V. A. Ho, M. Makikawa and S. Hirai, "Flexible Fabric Sensor toward a Humanoid Robotic Skin: Fabrication, Characterization, and Perceptions," IEEE Sensors Journal, vol. 13, No. 10, pp 4065-4080, 2013.

[20] V. A. Ho, S. Hirai and K. Naraki, "Fabric interface with proximity and tactile sensation for human-robot interaction," in Proceeding IEEE/RSJ International Conference on Intelligent Robots and Systems, pp 238245, Korea, Oct. 9-14, 2016. 